\title{
Convolutional neural networks approach for multimodal biometric identification system using the fusion of fingerprint, finger-vein and face images
}

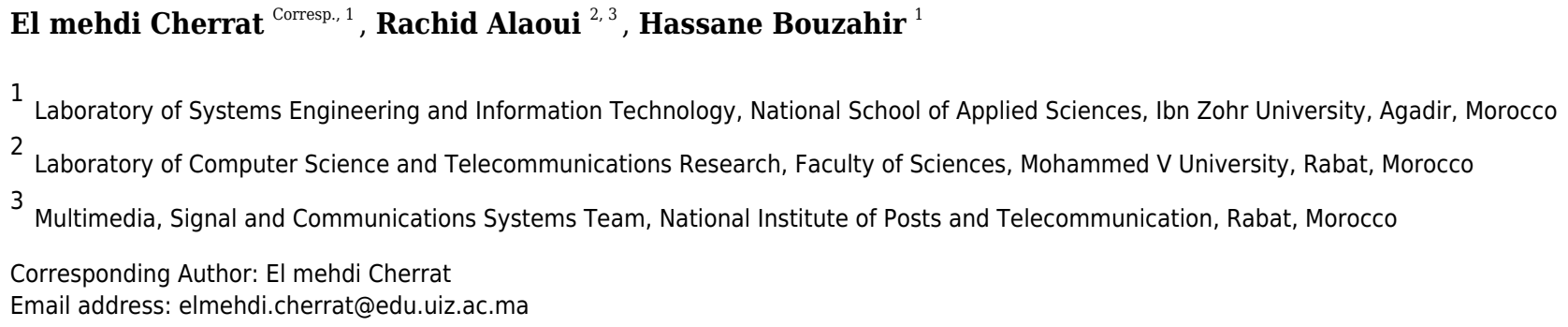

In recent years, the need for security of personal data is becoming progressively important. In this regard, the identification system based on fusion of multibiometric is most recommended for significantly improving and achieving the high performance accuracy. The main purpose of this paper is to propose a hybrid system of combining the effect of tree efficient models: Convolutional Neural Network (CNN), Softmax and Random Forest classifier based on multi-biometric fingerprint, finger-vein and face identification system. In conventional fingerprint system, image pre-processed is applied to separate the foreground and background region based on K-means and DBSCAN algorithm.

Furthermore, the features are extracted using CNNs and dropout approach, after that, the Softmax performs as a recognizer. In conventional fingervein system, the Region of interest (ROI) image contrast enhancement using exposure fusion framework is input into the CNNs model. Moreover, the Random Forest (RF) classifier is proposed for classification. In conventional face system, the CNNs architecture and Softmax are required to generate face feature vectors and classify personal recognition. The score provided by these systems is combined for improving Human identification. The proposed algorithm is evaluated on publicly available SDUMLA-HMT real multimodal biometric database using a GPU based implementation. Experimental results on the datasets has shown significant capability for identification biometric system. The proposed work can offer an accurate and efficient matching compared with other system based on unimodal, bimodal, multimodal characteristics. 
1 Convolutional Neural Networks Approach for

2 Multimodal Biometric Identification System Using the

3 Fusion of Fingerprint, Finger-Vein and Face Images

${ }^{1}$ Laboratory of Systems Engineering and Information Technology, National School of Applied

8 Sciences, Ibn Zohr University, Agadir, Morocco

$9{ }^{2}$ Laboratory of Computer Science and Telecommunications Research, Faculty of Sciences, Mohammed V University, Rabat, Morocco Telecommunication, Rabat, Morocco

Corresponding Author:

El mehdi Cherrat

\section{Abstract}

In recent years, the need for security of personal data is becoming progressively important. In this regard, the identification system based on fusion of multibiometric is most recommended for significantly improving and achieving the high performance accuracy. The main purpose of this paper is to propose a hybrid system of combining the effect of tree efficient models: Convolutional Neural Network (CNN), Softmax and Random Forest classifier based on multi-biometric fingerprint, finger-vein and face identification system. In conventional fingerprint system, image pre-processed is applied to separate the foreground and background region based on K-means and DBSCAN algorithm. Furthermore, the features are extracted using CNNs and dropout approach, after that, the Softmax performs as a recognizer. In conventional fingervein system, the Region of interest (ROI) image contrast enhancement using exposure fusion framework is input into the CNNs model. Moreover, the Random Forest (RF) classifier is proposed for classification. In conventional face system, the CNNs architecture and Softmax are required to generate face feature vectors and classify personal recognition. The score provided by these systems is combined for improving Human identification. The proposed algorithm is evaluated on publicly available SDUMLA-HMT real multimodal biometric database using a GPU based implementation. Experimental results on the datasets has shown significant capability for identification biometric system based on unimodal, bimodal, multimodal characteristics. 


\section{Introduction}

40 Biometric authentication system is basically a pattern-recognition system that identifies a human using a feature vector involved in a particular measurable morphological or behavioral characteristic the individual acquires. The biometrics modalities are often unique, measurable or automatically validated or permanent (Cherrat et al., 2017).

Fingerprint have become an essential biometric trait due to its uniqueness and invariant to every individual. This biometric modality is more used and acceptable by the users due to acquiring device is comparatively small. Moreover, the recognition accuracy is relatively very high to others biometric recognition system based on the retina, ear shape, iris, etc. (Borra et al., 2018). The finger vein biometric modality is usually used in biometric recognition because of many advantages compared other modality, 1) it is simple and easy to use : easily acquired using sensor capable of capturing or the NIR (Near-Infrared) light source; 2) it is high security: the vein structure is hidden inside the skin and the possibility of spoof the human recognition system is very complex; 3) the veins of each individual are unique and different (Yang \& Zhang, 2012). The fingervein recognition is based on human veins characteristic for identification or verification of the individual. As result, human and computer performance on fingervein recognition is a studies topic with both scientific research value and widely application prospects (Kang et al., 2019).

Face recognition is a biometric recognition technology based on human facial feature information for identification or verification. The algorithms using facial recognition are sensitive to variance in facial expressions and accessories, uncontrolled illumination, poses. In this regard, Human and computer performance on facial identification is a research topic with both scientific research value and widely application prospects (Mane $\&$ Shah, 2019).

In order to overcome the limitation concerned with the system based on one modality biometric, the multimodal biometric system increase the robustness and performance against the imposters attack and environment variations. This system is classified as multi-instance, multi-sensor, multialgorithm, multi-modal and hybrid systems (Walia et al., 2019). acquisition of biometric trait is process of getting a digitalized image of a person using specific capturing device. Second, the pre-processing is allowed to improve overall quality of the captured image. Third, the features data are extracted using different algorithms. Finally, the matching of the extracted characteristics is generally applied in order to perform the recognition of the individual.

The multi-biometric recognition system combines a variety of biometric sources. The main advantage of multimodal system against traditional single biometric is achieving the recognition process more secure and accurate (Unar et al., 2014). In this regard, researches of multimodal biometric using finger-vein and face images are prevalent and essential recently.

The advantage of combining the fingerprint, finger veins and face is the ability to establish an image acquisition system which can capture fingerprint and finger-vein images simultaneously (they find in almost at the same place) and its devices are less expensive and easier to deploy. 
79 Moreover, the face is one of the most natural methods to identify an individual, it does not restrict

80 the movement of the person and its deployment cost is relatively low.

81 The proposed method deploys the multimodal biometric recognition system that is combined the

82 fingerprint, finger-vein and face images using Convolutional Neural Networks (CNNs)

83 architectures and classifiers based on Softmax and Random Forest (RF). Our scheme is efficient

84 to various environmental changes and database types. Figure 1 describes general block diagram of

85 the proposed recognition system.

86 The rest of the paper is separated as follows: Section 2 defines a concise description of related

87 research. Section 3 refers to the processes that are part of our proposed algorithm. While Section

884 elucidates the experimental results, Section 5 concludes the proposed work.

89

90

91

92

93

94

95

96

97

98

99

100

101

102

103

104

105

106

107

108

109

110

111

112

113

114

115

116

117

\section{Literature review}

Many studies has been conducted to investigate multimodal biometric system and its effects on the human recognition. Ross et al (Ross \& Jain, 2003) presented different levels of fusion and score level fusion on the multimodal biometric system (fingerprint, face, voice and hand geometry) using the sum rule. However, this method needs to conduct experiments on a larger database of users for recognition system. Yang.al (Yang \& Zhang, 2012) have been subjected a fusion of fingerprint and finger vein. These biometric characteristic is extracted using a unified Gabor filter method. The feature level fusion is generated based on supervised local preserving canonical correlation analysis framework. For individual identification, the nearest neighborhood classifier is applied. However, the performance is evaluated using a collected database which contains just 640 fingerprint images and 640 finger-vein images. Son.al (Son \& Lee, 2005) have been subjected a fusion of face and iris using DWT and DLDA method. Each experiment is repeated at least 20 times for reducing the variation. Though, this algorithm is not compared with other state of the art methods. As well, it is not verified on a large number of data. Ross.al (Ross \& Govindarajan, 2005) presented multimodal biometric system that uses hand and face at feature level for biometric recognition purposes. Moreover, the experiments have been tested on intra-modal and inter-modal fusion with R, G, B channels. The drawbacks of this system, it does not accord incompatible feature sets (for example eigen-coefficients of face and minutiae points of fingerprints) to be combined and it is difficult to predict the best fusion strategy given a scenario. A novel fingerprint and finger vein identification system by concatenating the feature vectors are achieved by Ma.al (Ma, Popoola \& Sun, 2015). In this study, the extracted feature vectors of both fingerprint and finger vein images are concatenated in order to combine the classifiers recognition results at the decision level. Though, the accuracy of this technique does not satisfy the requirement of many real-world applications, where it suffers from significant performance translation and rotation invariant. Huang et al (Huang et al., 2015) introduced an adaptive bimodal sparse representation based on classification, i.e. adaptive face and ear using bimodal recognition system based on sparse coding, where the qualities of weighted feature is selected. This system requires to pre-process each trait biometric before extracting the features. Furthermore, the recognition accuracy needs to increase. 
118 Yang et al. (Yang et al., 2018) presented a multi-biometric system cancelable using fingerprint 119 and finger-vein, which combines the minutia points of fingerprint and finger-vein image feature 120 based on a feature-level of three fusion techniques. However, the effect of noisy data on the 121 performance of the system is not included. Vishi. al (Vishi \& Mavroeidis, 2018) reported a fusion 122 of fingerprint and finger-vein for identification using the combinations of score normalization 123 (min-max, z-score, hyperbolic tangent) and fusion methods (minimum score, maximum score,

124

125

126

127

128

129

130

131

132

133

134

135

136

137

138

139

140

141

142

143

144

145

146

147

148

149

150

151

152

153

154

155

156 simple sum, user weighting). The pre-processing stage is not used in this algorithm. Thus, the recognition accuracy can be decreased. Jain.al (Jain et al., 1999) introduced a multimodal biometric system using face, fingerprint, and voice. Moreover, the different fusion techniques and normalization methods of fingerprint, hand geometry and face biometric sources are achieved by Jain.al (Jain, Nandakumar \& Ross, 2005). The drawback of these methods, they need to be tested on a large dataset in real operating environment. Soleymani.al (Soleymani et al., 2018) are suggested a multimodal biometric system with face, iris, and fingerprint using multiple streams of modality-specific CNNs. In this algorithm, some complexity also exists in multimodal recognition system which reduces its acceptability in many areas. Further, multimodal biometric system based on Iris, finger vein and fingerprint was investigated (Walia et al, 2019). In this method, individual classifier score estimation along with its performance optimization using evolutionary Backtracking Search Optimization Algorithm (BSA) is presented. In addition, the core design of the fusion model using proportional conflict redistribution rules (PCR-6) is proposed. The accuracy of $98.43 \%$ and an error rate of $1.57 \%$ have been achieved. However, the enhancement biometric quality and Experimentation with real multimodal dataset is not used in this system.

There exist only a few works about a multimodal biometric system that includes fingerprint, fingervein and face. Rajesh.al (Rajesh \& Selvarajan, 2017) are proposed an algorithm for biometric recognition using fingerprint and fingervein and face. They used score-level fusion to fuse these biometrics traits but they did not evaluate such a system against to others methods. As well, they have not provided information on the databases and the number of users in the study.

\section{Proposed system}

\section{Fingerprint Recognition System}

This section describes the detail about the proposed fingerprint recognition system using CNNSoftmax. In this work, our proposed method includes of the following three major stages: (1) preprocessing the fingerprint image; (2) feature extraction with CNN model; (3) using Softmax as a classifier. For pre-processing step, Soble and TopHat filtering method improved the quality of the image by limiting the contrast. After that, K-means and DBSCAN approaches are applied to classify the image into foreground and background region (Cherrat, Alaoui \& Bouzahir, 2019). In addition, the Canny method (Canny, 1987) and the inner rectangle are adopted to extract the ROI of fingerprint segmented. After this step, the features are extracted from the pre-processing fingerprint image using the $\mathrm{CNN}$ architecture.

CNN is a convolutional neural network based on deep supervised learning model. In this regard, $\mathrm{CNN}$ can be viewed an automatic feature extractor and a trainable classifier (Bhanu, \& Kumar, 
157 2017). As shown in Fig. 2, the configuration details of the proposed fingerprint-CNN architecture.

158 The proposed model has 5 convolutional layers and 3 max-pooling layers which can be are

159 computed using equation 1. In addition, 3 Rectified Linear Unit (ReLU) are used to our system 160 which can be defined as equation 2.

$161 O_{n}=\sum_{i=1}^{N-1} x_{i} f_{n-i}$

162 where $\mathrm{O}$ is the output map, $\mathrm{x}$ is input map, $\mathrm{f}$ is the filter and $\mathrm{N}$ is number of elements in $\mathrm{x}$.

$163 f(x)=\max (0, x)$

164 where $\mathrm{x}$ is the input to a neuron.

165 The Softmax function can be used to the fully convolutional layer output, as shown in equation 3.

$166 S(r, i)=-\log \left(\frac{e^{z_{i}}}{\sum_{k=1}^{N} e^{z_{j}}}\right)$

167

168

169

170

171

172

173

174

175

176

177

178

179

180

181

182

183

184

185

186

187

188

189

190

191

when the vector of output neurons is set to $r$, the probability of the neurons appropriate to the $\mathrm{i}^{\text {th }}$ class is provided by separation the value of the $\mathrm{i}^{\text {th }}(\mathrm{i}=1 \ldots \mathrm{j})$ element by the sum of the values of all elements.

The structure is described as follows : 1) L1 : the input layer data size of $88 \times 88$, which is the size of input pre-processing fingerprint images ; 2) L1M1: first hidden layer, composed by 64 convolutional filters of size $3 \times 3 \times 1$, ReLU activation function and a max-pooling layer of size $2 \times 2$. This layer changes the input data into CL1M1 $=[42 \times 42 \times 64]$ features ; 3) L2M2: second hidden layer, composed by 128 convolutional filters of size $3 \times 3 \times 64$, ReLU activation function and a max-pooling layer of size $2 \times 2$. This layer changes the input data into CL2M2 $=[19 \times 19 \times 128]$ features; 4) L3M3: third hidden layer, composed by 128 convolutional filter of size $3 \times 3 \times 128$, ReLU activation function and a max-pooling layer of size $2 \times 2$. In order to disconnect the connections between the first layer and the next layers the dropout probability [19] of $20 \%$ is adopted. This layer transforms the input data into CL3M3 $=[9 \times 9 \times 256]$ features ; 5) L4M4: forth hidden layer namely fully connected layer (FCL), represented the flattening process, which is converted all the resultant two-dimensional arrays into a single long continuous linear vector. The features size of input data is $1 \times 1 \times 20736$; 6) L5M5: final hidden layer, this layer represented the feature descriptor of the finger vein for recognition to describe it with informative features. The Softmax function is used to predict labels of the input patterns.

\section{Fingervein Recognition System}

In this section, the proposed algorithm for fingervein recognition using $\mathrm{CNN}$ as a feature extractor is described. Our proposed method consist in three phases: 1) Canny method and the inner rectangle are used to obtain the ROI of finger vein image; 2) the exposure fusion framework (EFF) (Ying, et al., 2017) is applied to improve the contrast of the image by limiting the contrast amplification in the different region of the image. The result of finger vein image using Canny edge detector and contrast techniques such as contrast limited adaptive histogram equalization 
192 (CLAHE) (Reza, 2004) and dynamic histogram equalization (DHE) (Abdullah-Al-Wadud et al., 193 2007) is shown in Fig. 3. 3) feature extraction based on CNN and 4) RF is employed as a classifier 194 for fingervein classification. The proposed model has 5 convolutional layers where 3 followed by 195 max-pooling and 3 Rectified Linear Unit (ReLU). The RF classifier is used to predict labels of the 196 input patterns. Table 1 summarizes the characteristics of the proposed fingervein-CNN 197 configuration.

198

199

\section{Random Forest Classifier}

200

201

202

203

204

205

206

207

208

209

210

211

212

213

214

215

216

\section{7}

218

219

220

221

222

223

224

225

226

The Random forest (RF) algorithm proposed by Breiman (Breiman, L. 2001), is an ensemble learning technique for regression and classification. At each split, RF consists of bagging (bootstrap aggregating) of $\mathrm{T}$ decision trees with a randomized selection of features. Given a training data X, the RF algorithm is presented as follows: (i) at each T, generate a bootstrap sample with replacement from the original training data. (ii) Choose a random set of features using each bootstrap sample data, at each internal node. Furthermore, randomly select $Y$ predictors and pick the best split based on only the Y predictors rather than all predictors. (iii) Aggregate the set of estimated decision trees in order to get a single one.

\section{Face Recognition System}

In this section, the proposed algorithm for face recognition using CNN as a feature extractor is described. Our proposed method consist in two phases: feature extraction based on CNN and employing Softmax as a classifier for face classification. Table 2 shows the configuration details of the proposed $\mathrm{CNN}$ architecture using face image. The proposed model has 5 convolutional layers where 3 followed by max-pooling and 3 Rectified Linear Unit (ReLU). In order to disconnect the connections between the first layer and the next layers the dropout probability (Srivastava et al., 2014) of $20 \%$ is adopted. In addition, the dropout probability of $10 \%$ between the second layer and the next layers.

\section{Feature Extraction Fusion}

In this section, we introduce our proposed method score level fusion technique based on the matching score level fusion. This matching score indicates better proximity of characteristic vector with the template.

The fused score level is based on the weighted sum and weighted product as shown in equations (4)-(5). If the fused score value providing of the query fingerprint, finger vein and face is greater than or equal to the decision threshold value. Then, the person is accepted, otherwise is rejected (Singh, Singh \& Ross,. 2019).

Score $_{w s}=w_{1} S_{F P}+w_{2} S_{F V}+w_{3} S_{F A}$

Score $_{w p}=S_{F P}{ }^{w_{1}} \times S_{F V}{ }^{w_{2}} \times S_{F A}{ }^{w_{3}}$ 
227 where $S_{F P}, S_{F V}, S_{F A}$ indicate the scores of biometric matcher, $w_{1}, w_{2}, w_{3}$ are the weight value 228 over a range of $[0,1]$ and according to sum of $w_{1}, w_{2}, w_{3}$ is always 1 .

\section{Data augmentation}

230 Data augmentation is one of the methods for reducing the effects of overfitting problems in CNN

231

232

233

234

235

236

\section{7}

238

239

240

241

242

243

244

245

246

247

248

249

250

251

252

253

254

255

256

257

258

259

260

261

262

263

architecture. This technique is employed to increase the amount of training data based on image translation, rotation and cropping process. Many previous works have been successfully used dataaugmentation method. We implemented the data augmentation as expand to the work in (Krizhevsky et al., 2012) such as the rotation and the translation (left, right, up and down) (Park et al., 2016). For SDUMLA-HMT database augmentation, we were augmented that is 2 times larger than the original database.

\section{Experimental results and discussion}

The experimental operation platform in this study is described as follows: the host configuration: Intel Core i7 - 4770 processor, 8Go RAM and NVIDIA GeForce GTX 980 4GO GPU, runtime environment: Ubuntu 14.04 LTS (64 bit). In order to better verify our algorithm, the following classification methods are adopted in the experiment: Support Vector Machine (SVM) (Cortes \& Vapnik, 1995), Random Forest (RF) (Breiman, L. 2001), Logistic Regression (LR) (Hosmer et al., 2013), fingervein biometric system (Itqan, et al., 2017) and Multimodal biometric system using fingerprint, fingervein and face (Rajesh \& Selvarajan, 2017). These algorithms were compared to each other. In order to validate the proposed algorithm, the results have been tested on the public on SDUMLA-HMT (Yin, et al., 2011) database which includes real multimodal data of fingerprint, fingervein and face images. The total number of training images was 41340 and we divided them into training, validation, and test sets. The divided data set used in the experiment is shown in Table 3.

The performance measure is accuracy rate as defined by Equation 9 .

Accuracy $=\frac{T P+T N}{T P+T N+F P+F N} \times 100$

where TP (True Positive Rate) is the probability of authorized users that are recognized correctly over the total number tested, TN (True Negative Rate) is the probability of authorized users that are not recognized over the total number tested. FP (False Positive Rate) describes the percentage of unauthorized users that are recognized to the total number tested. FN (False Negative Rate) describes the percentage of unauthorized users that are not recognized falsely to the total number tested.

As can be seen from Table 4, the proposed fingerprint recognition using CNN with dropout method leads to a significant performance improvement on real database multimodal.

In particular, the highest accuracy gain was obtained by dropout method on four datasets for training and test set. Moreover, the least loss is achieved based on database using the dropout method. For training set, it can be noted from the Table 4 that the accuracy of $99.13 \%$ is augmented to $99.63 \%$ and the lost rate of $2.16 \%$ is reduced to $1.17 \%$ in the proposed method due to add the 
264 drop function in our system. For test set, based on the results yielded in Table 5, the accuracy of $26599.33 \%$ is augmented to $99.48 \%$ and the lost rate of $2.16 \%$ to $2.03 \%$ in the proposed fingerprint 266 identification method.

267 The comparison of pre-processed algorithms with the proposed fingervein system are shown in 268 Table 6 and Table7. The proposed work gives the highest average accuracy for training set with $26999.09 \%$ and least loss with $2.69 \%$. For test set, also the highest average accuracy of $99.27 \%$ and 270 least loss with 2.05 is obtained using our algorithm.

271 The results of Table 8 and Table 9 show that dropout method plays an important role in increasing 272 the accuracy of the proposed face recognition system. For training set, the accuracy of $99.25 \%$ is 273 augmented to $99.55 \%$ and the lost rate of $1.96 \%$ is lowered to $1.77 \%$. For test set, the accuracy of $27499.05 \%$ is augmented to $99.13 \%$ and the lost rate of $2.10 \%$ is lowered to $2.17 \%$.

275 From Table 10, we compared the results using the SVM, LR, RF with CNN using the proposed 276 fingerprint system to show the highest accuracy of $99.48 \%$ is tacked using the Softmax classifier. 277 As shown in this Table, the RF classifier gives the highest accuracy of $99.53 \%$ using the proposed 278 fingervein system using CNN architecture. Also based on the results yielded in Table 10 it can be 279 argued that the Softmax classifier gives the highest accuracy of $99.13 \%$ based on the proposed 280 face system using CNN model.

281 From Table 11, it is clear that the highest recognition rate is obtained when weighted sum is used 282 for fusion rule. Table 12 presents computational time for fusion method of database. Table 13 283 shows the comparison between the proposed system unimodal, bimodal and multimodal biometric 284 that using $\mathrm{CNN}$ on database. The proposed fingerprint, fingervein and face as a bimodal system 285 can be used for recognition with acceptable identification results comparing with other unimodal 286 systems. The proposed multimodal biometric system is increased the recognition accuracy than 287 the unimodal and bimodal identification system, where the accuracy rate is $99.49 \%$. Although 288 existing biometric method (Walia et al., 2019) is able to obtain $99.61 \%$ of recognition rate, it is 289 still slower than our proposed fusion method in terms of computational time of $69 \mathrm{~ms}$.

290 Finally, we can conclude from these results that the proposed multimodal system is superior to 291 other methods because:

292 1) The proposed enhanced fingerprint and finger vein patterns are significantly clearly 293 distinguishable and more prominent in their others enhanced versions. Therefore, the proposed 294 methods are typically able to guarantee a high identification rate.

295 2) The recognition accuracy based on dropout method is better than using only the dataset method.

296 3) CNN approach can usually provide better performances than using combinations between 297 different processes such as windowing, extracting features, etc. Thus, the recognition biometric 298 system based on CNN technique can surpassed other classical and complicated techniques.

299 4) The proposed multimodal algorithm have higher accuracy to identify the person and ensure that 300 its information or data is safer compared to system based on single or bimodal biometrics. 
301

302

303

304

305

306

307

308

309

310

311

312

313

314

315

316

317

318

319

320

321

322

323

324

325

326

327

328

329

330

331

332

333

334

335

336

337

338

339

\section{Conclusions}

A system for Human recognition used Convolutional Neural Networks (CNN) model and multimodal biometric identification system based on the fusion of fingerprint, fingervein and face images has been introduced in this work. The experimental results on real multimodal database has shown that the overall performance of the proposed multimodal system is better than unimodal and bimodal biometric systems based on $\mathrm{CNN}$ and different classifiers regarding identification. From the results obtained, we can also conclude that the effect of pre-processed algorithm is improved the accuracy rate of the proposed system. Dropout technique plays an important role for increasing the recognition accuracy, which reduced the loss rate of the system. For future study, extending the proposed algorithm to other applications is a task worth investigating. Where, it will be tested with a more challenging dataset that contains a large number of subjects.

\section{References}

Cherrat, E. M, Alaoui, R, Bouzahir, H, Jenkal, W. 2017. High-density salt-and-pepper noise suppression using adaptive dual threshold decision based algorithm in fingerprint images. In 2017 Intelligent Systems and Computer Vision (ISCV) IEEE: 1-4.

\section{Borra, S. R, Reddy, G. J, Reddy, E. S. 2018. An Efficient Fingerprint Identification using} Neural Network and BAT Algorithm. International Journal of Electrical \& Computer Engineering, 8:2.

Yang, J, Zhang, X. 2012. Feature-level fusion of fingerprint and finger-vein for personal identification. Pattern Recognition Letters, 33(5):623-628.

Kang, W, Lu, Y, Li, D, Jia, W. 2019. From Noise to Feature: Exploiting Intensity Distribution as a Novel Soft Biometric Trait for Finger Vein Recognition. IEEE Transactions on Information Forensics and Security, 14(4): 858-869.

Mane, S, Shah, G. 2019. Facial Recognition, Expression Recognition, and Gender Identification. In Data Management, Analytics and Innovation Springer, Singapore: 275-290. Walia, G. S, Rishi, S., Asthana, R., Kumar, A., \& Gupta, A. 2019. Robust Secure multimodal biometric system based on diffused graphs and optimal score fusion. IET Biometrics, 8(4), 231242.

Unar, J. A., Seng, W. C., \& Abbasi, A. 2014. A review of biometric technology along with trends and prospects. Pattern recognition, 47(8), 2673-2688.

Ross, A, Jain, A. 2003. Information fusion in biometrics. Pattern recognition letters, 24(13), 21152125.

Son, B, Lee, Y. 2005. Biometric authentication system using reduced joint feature vector of iris and face. In International Conference on Audio-and Video-Based Biometric Person Authentication Springer, Berlin, Heidelberg: 513-522.

Ross, A. A, Govindarajan, R. 2005. Feature level fusion of hand and face biometrics. In Biometric Technology for Human Identification II. International Society for Optics and Photonics. (5779):196-205. 
340 Ma, H, Popoola, O. P, \& Sun, S. 2015. Research of dual-modal decision level fusion for 341 fingerprint and finger vein image. International Journal of Biometrics, 7(3): 271-285.

342 Huang, Z., Liu, Y., Li, X., \& Li, J. 2015. An adaptive bimodal recognition framework using 343 sparse coding for face and ear. Pattern Recognition Letters, 53, 69-76.

344 Yang, W, Wang, S, Hu, J, Zheng, G, Valli, C. 2018. A fingerprint and finger-vein based 345 cancelable multi-biometric system. Pattern Recognition, 78:242-251.

346 Vishi, K, Mavroeidis, V. 2018. An evaluation of score level fusion approaches for fingerprint and 347 finger-vein biometrics. arXiv preprint arXiv:1805.10666.

348 Jain, A. K, Hong, L, \& Kulkarni, Y. 1999. A multimodal biometric system using fingerprint, 349 face and speech. In Second Int'l Conf. AVBPA: 10.

350 Jain, A, Nandakumar, K, Ross, A. 2005. Score normalization in multimodal biometric systems. 351 Pattern recognition, 38(12):2270-2285.

352 Soleymani, S, Dabouei, A, Kazemi, H, Dawson, J, Nasrabadi, N. M. 2018. Multi-level feature 353 abstraction from convolutional neural networks for multimodal biometric identification. In 2018

354

355

356

357

358

359

360

361

362

363

364

365

366

367

368

369

370

371

372

373

374

375

376

377

378 24th International Conference on Pattern Recognition (ICPR) IEEE: 3469-3476.

Rajesh, S., \& Selvarajan, S. 2017. Score Level Fusion Techniques in Multimodal Biometric System Using CBO-ANN. Research Journal of Biotechnology. Vol. 12 (Special Issue II):79-87.

Cherrat, E. M, Alaoui, R, Bouzahir. 2019. Improving of fingerprint segmentation images based on K-means and DBSCAN clustering. International Journal of Electrical \& Computer Engineering 9(4): 2425-2432.

Canny, J. 1987. A computational approach to edge detection. In Readings in computer vision. Morgan Kaufmann: 184-203.

Bhanu, B, Kumar, A. Eds. 2017. Deep learning for biometrics. Springer.

Ying, Z, Li, G, Ren, Y, Wang, R, Wang, W. 2017. A new image contrast enhancement algorithm using exposure fusion framework. In International Conference on Computer Analysis of Images and Patterns. Springer, Cham: 36-46.

Reza, A. M. 2004. Realization of the contrast limited adaptive histogram equalization (CLAHE) for real-time image enhancement. Journal of VLSI signal processing systems for signal, image and video technology, 38(1): 35-44.

Abdullah-Al-Wadud, M, Kabir, M. H, Dewan, M. A. A, Chae, O. 2007. A dynamic histogram equalization for image contrast enhancement. IEEE Transactions on Consumer Electronics, 53(2): 593-600.

Breiman, L. 2001. Random forests. Machine learning, 45(1):5-32.

Srivastava, N, Hinton, G., Krizhevsky, A, Sutskever, I, Salakhutdinov, R. 2014. Dropout: a simple way to prevent neural networks from overfitting. The Journal of Machine Learning Research, 15(1): 1929-1958.

Park, E, Kim, W, Li, Q, Kim, J, Kim, H. 2016. Fingerprint liveness detection using CNN features of random sample patches. In International Conference of the Biometrics Special Interest Group (BIOSIG) IEEE: 1-4. 
379 Krizhevsky, A, Sutskever, I, Hinton, G. E. 2012. Imagenet classification with deep 380 convolutional neural networks. In Advances in neural information processing systems: 1097-1105. 381 Singh, M, Singh, R, Ross, A. 2019. A Comprehensive Overview of Biometric Fusion. Information 382 Fusion.

383 Cortes, C, Vapnik, V. 1995. Support-vector networks. Machine learning, 20(3): 273-297.

384 Hosmer Jr, D. W, Lemeshow, S, Sturdivant, R. X. 2013. Applied logistic regression (398). John 385 Wiley \& Sons.

386 Tome, P, Vanoni, M, Marcel, S. 2014. On the vulnerability of finger vein recognition to spoofing. 387 In 2014 International Conference of the Biometrics Special Interest Group (BIOSIG) IEEE: 1-10. 388 Yin, Y, Liu, L, \& Sun, X. (2011) SDUMLA-HMT: a multimodal biometric database. In : Chinese 389 Conference on Biometric Recognition. Springer, Berlin, Heidelberg. p. 260-268.

390 Itqan, K. S., Syafeeza, A. R., Gong, F. G., Mustafa, N., Wong, Y. C., \& Ibrahim, M. M. (2016). 391 User identification system based on finger-vein patterns using Convolutional Neural 392 Network. ARPN Journal of Engineering and Applied Sciences, 11(5), 3316-3319. 
Figure 1

General block diagram of the proposed recognition system

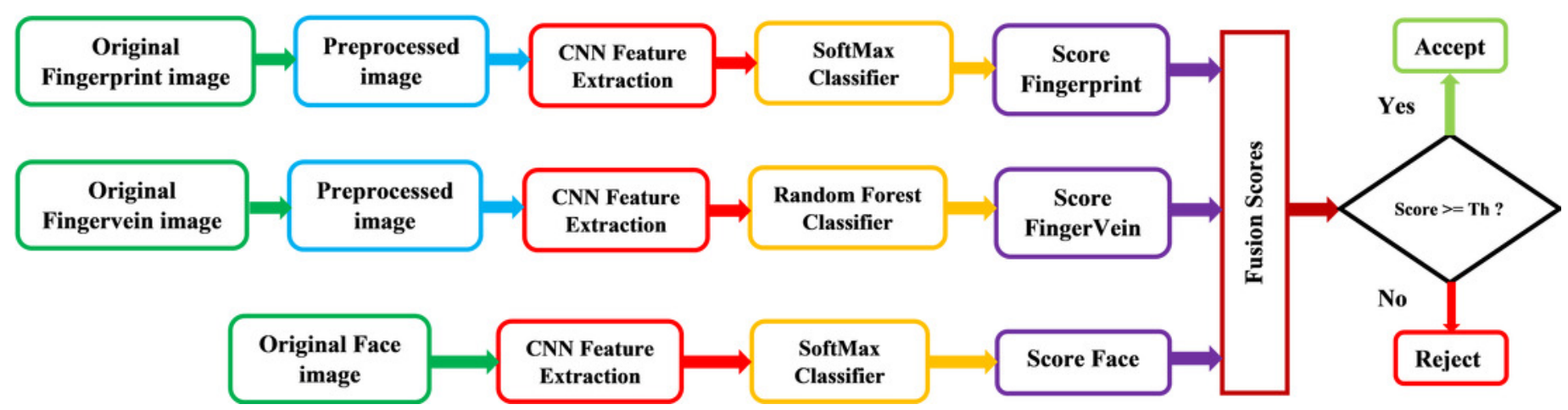


Figure 2

The architecture of the proposed Fingerprint-CNN model

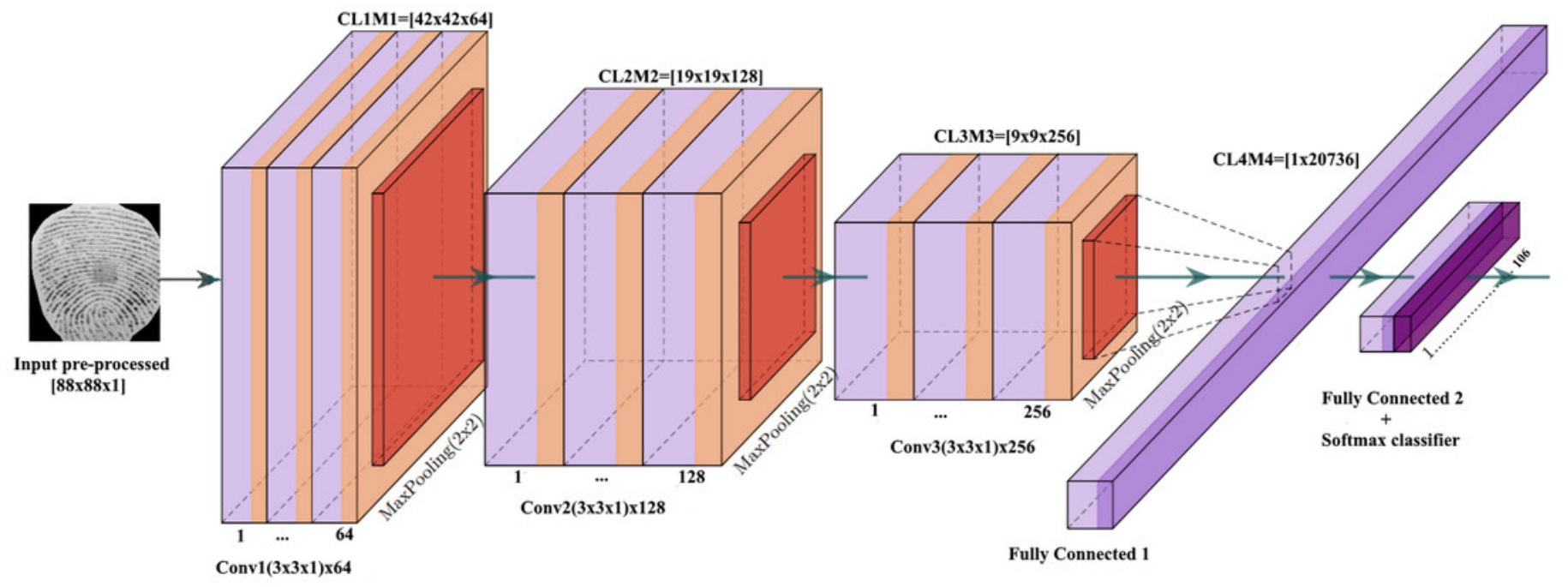




\section{Figure 3}

Pre-processed finger-vein image using different enhanced algorithm from the Avera databases

$(A),(F),(K),(Q)$ Original image. (B), (G), (L), (R) Cropped image. (C), (H), (M), (S) CLAHE enhanced. (D), (I), (N), (T) DHE enhanced. (E), (J), (O), (U) Proposed enhanced using EFF.
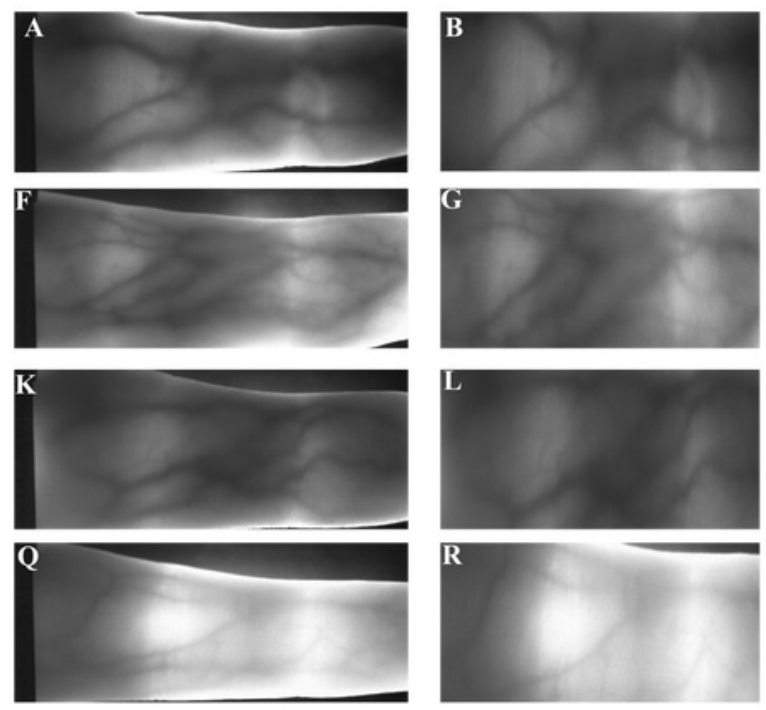
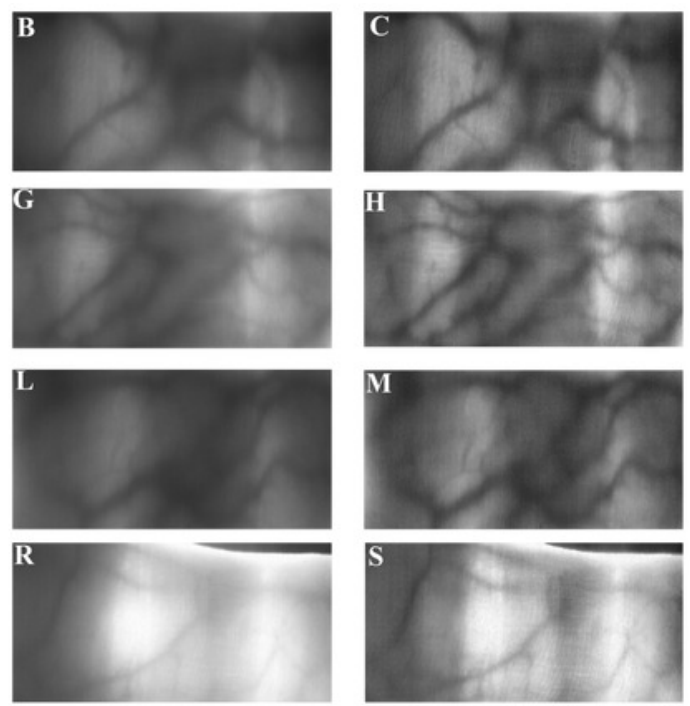
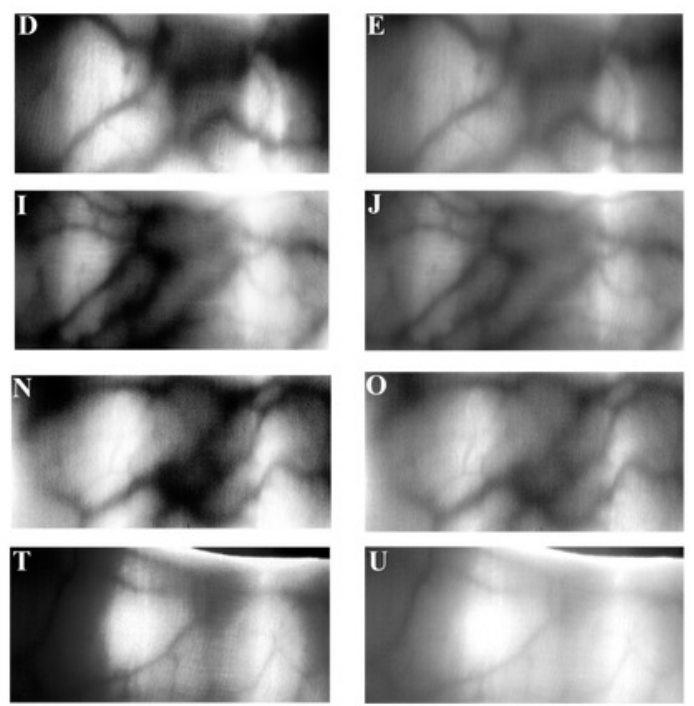


\section{Table 1 (on next page)}

Proposed Fingervein-CNN configuration 


\begin{tabular}{cccc} 
Type & Number of Filter & Size of Feature Map & Filter size / stride \\
\hline Convolution & 64 & $58 \times 150 \times 1$ & $3 \times 3 / 1$ \\
ReLU & - & $58 \times 150 \times 1$ & - \\
Max-Pooling & 1 & $29 \times 75 \times 64$ & $3 \times 3 / 2$ \\
Convolution & 128 & $27 \times 73 \times 128$ & $3 \times 3 / 1$ \\
ReLU & - & $27 \times 73 \times 128$ & - \\
Max-Pooling & 1 & $13 \times 36 \times 128$ & $3 \times 3 / 2$ \\
Convolution & 256 & $11 \times 34 \times 256$ & $3 \times 3 / 1$ \\
ReLU & - & $11 \times 34 \times 256$ & - \\
Max-Pooling & 1 & $5 \times 17 \times 256$ & $3 \times 3 / 2$ \\
Fully-connected & 1 & $1 \times 21760$ & - \\
Fully-connected & 1 & $1 \times 106$ & - \\
\hline
\end{tabular}

1 


\section{Table 2 (on next page)}

Proposed Face-CNN configuration 


\begin{tabular}{cccc} 
Type & Number of Filter & Size of Feature Map & Filter size / stride \\
\hline Convolution & 32 & $88 \times 88 \times 1$ & $3 \times 3 / 1$ \\
ReLU & - & $88 \times 88 \times 1$ & - \\
Max-Pooling & 1 & $44 \times 44 \times 32$ & $3 \times 3 / 2$ \\
Convolution & 64 & $42 \times 42 \times 64$ & $3 \times 3 / 1$ \\
ReLU & - & $42 \times 42 \times 64$ & - \\
Max-Pooling & 1 & $21 \times 21 \times 64$ & $3 \times 3 / 2$ \\
Convolution & 128 & $19 \times 19 \times 128$ & $3 \times 3 / 1$ \\
ReLU & - & $19 \times 19 \times 128$ & - \\
Max-Pooling & 1 & $9 \times 9 \times 128$ & $3 \times 3 / 2$ \\
Fully-connected & 1 & $1 \times 10388$ & - \\
Fully-connected & 1 & $1 \times 106$ & - \\
\hline
\end{tabular}

1 


\section{Table 3 (on next page)}

Dataset structure of fingerprint, fingervein and face databases 


\section{SDUMLA-HMT Database}

\begin{tabular}{cc}
\hline Class Number & 106 \\
Image Number & 41340 \\
Training & 33072 \\
Validation & $4134 \square$ \\
Test & 4134 \\
\hline
\end{tabular}

1 


\section{Table 4 (on next page)}

The trianing set result of proposed fingerprint recognition using CNN 


\begin{tabular}{ccccc} 
& \multicolumn{2}{c}{ Train Set Without Dropout } & \multicolumn{2}{c}{ Training Set With Dropout } \\
Images & Accuracy (\%) & Loss (\%) & Accuracy (\%) & Loss (\%) \\
\hline Original $_{\mathrm{FP}}$ & 98.96 & 3.65 & 99.31 & 2.35 \\
Enhanced $_{\mathrm{FP}}$ & 99.49 & 1.93 & 99.56 & 1.23 \\
Proposed Enhanced $_{\mathrm{FP}}$ & 99.13 & 2.16 & 99.63 & 1.17 \\
\hline
\end{tabular}




\section{Table 5 (on next page)}

The test set result of proposed fingerprint recognition using CNN 


\begin{tabular}{ccccc} 
Images & Accuracy (\%) & Loss (\%) & Accuracy (\%) & Loss (\%) \\
\hline Original $_{\mathrm{FP}}$ & 97.06 & 9.12 & 97.66 & 5.71 \\
Enhanced $_{\mathrm{FP}}$ & 98.29 & 5.68 & 99.16 & 3.14 \\
Proposed Enhanced $_{\mathrm{FP}}$ & 99.33 & 2.16 & 99.48 & $\mathbf{2 . 0 3}$ \\
\hline
\end{tabular}




\section{Table 6 (on next page)}

The training set result of proposed fingervein recognition using CNN 
1

2

\begin{tabular}{ccc} 
Images & Accuracy (\%) & Loss (\%) \\
\hline Original $_{\mathrm{FV}}$ & 96.58 & 19.12 \\
Cropped $_{\mathrm{FV}}$ & 97.09 & 15.22 \\
CLAHE $_{\mathrm{FV}}$ & 98.45 & 6.36 \\
DHE $_{\mathrm{FV}}$ & 97.65 & 11.12 \\
Proposed Enhanced $_{\mathrm{FV}}$ & 99.09 & 2.69 \\
\hline
\end{tabular}




\section{Table 7 (on next page)}

The test set result of proposed fingervein recognition using CNN 
1

2

\begin{tabular}{ccc} 
Images & Accuracy (\%) & Loss (\%) \\
\hline Original $_{\mathrm{FV}}$ & 96.98 & $\mathbf{1 2 . 0 8}$ \\
Cropped $_{\mathrm{FV}}$ & 97.89 & 9.32 \\
CLAHE $_{\mathrm{FV}}$ & 98.97 & 4.23 \\
DHEE $_{\mathrm{FV}}$ & 98.25 & 5.52 \\
Proposed Enhanced $_{\mathrm{FV}}$ & 99.27 & $\mathbf{2 . 0 5}$ \\
\hline
\end{tabular}




\section{Table 8 (on next page)}

The training set result of proposed face recognition using CNN 
1

2

\begin{tabular}{ccccc} 
& \multicolumn{2}{c}{ Train Set Without Dropout } & \multicolumn{2}{c}{ Training Set With Dropout } \\
Images & Accuracy (\%) & Loss (\%) & Accuracy (\%) & Loss (\%) \\
\hline Original $_{\mathrm{Fa}}$ & $\mathbf{9 9 . 2 5}$ & 1.96 & 99.55 & 1.77 \\
\hline
\end{tabular}




\section{Table 9 (on next page)}

The test set result of proposed face recognition using CNN 
1

2

Test Set Without Dropout Test Set With Dropout

Images

Original $_{\mathrm{Fa}}$
Accuracy (\%)

Loss (\%)

Accuracy (\%) Loss (\%)

\begin{tabular}{ccccc} 
Images & Accuracy (\%) & Loss (\%) & Accuracy (\%) & Loss (\%) \\
\hline Original $_{\mathrm{Fa}}$ & 99.05 & 2.10 & 99.13 & 2.27 \\
\hline
\end{tabular}




\section{Table $\mathbf{1 0}$ (on next page)}

The result of proposed system recognition unimodal biometric using CNN with different classifiers 
1

\begin{tabular}{cccc} 
Classifiers & Fingerprint & Finger vein & Face \\
\hline & & & \\
CNN \& SoftMax & $99.48 \%$ & $99.27 \%$ & $99.13 \%$ \\
CNN \& SVM & $97.65 \%$ & $99.33 \%$ & $97.88 \%$ \\
CNN \& LR & $85.61 \%$ & $84.14 \%$ & $92.43 \%$ \\
CNN \& RF & $97.33 \%$ & $99.53 \%$ & $91.95 \%$ \\
\hline
\end{tabular}




\section{Table 11 (on next page)}

The result of proposed recognition systems using CNN with rules fusion 
1

\begin{tabular}{|c|c|c|}
\hline \multirow[t]{2}{*}{ Algorithms } & \multicolumn{2}{|c|}{ Rules fusion } \\
\hline & $\begin{array}{c}\text { Weighted } \\
\text { sum }\end{array}$ & $\begin{array}{c}\text { Weighted } \\
\text { product }\end{array}$ \\
\hline Fingerprint $_{\mathrm{CNN} \&}$ Fingervein $_{\mathrm{CNN}}$ & 99.59 & 99.58 \\
\hline Fingerprint $_{\mathrm{CNN}} \&$ Face $_{\mathrm{CNN}}$ & 99.30 & 99.28 \\
\hline Fingervein $_{\mathrm{CNN} \& \text { Face }_{\mathrm{CNN}}}$ & 99.20 & 99.17 \\
\hline Fingerprint $_{\mathrm{CNN}} \&$ Fingervein $_{\mathrm{CNN}} \&$ Face $_{\mathrm{CNN}}$ & 99.73 & 99.70 \\
\hline
\end{tabular}




\section{Table 12 (on next page)}

Computational time (ms) for fusion method of database. 
Algorithms

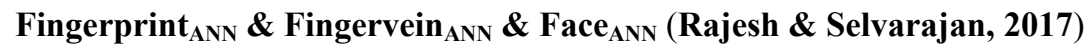

Score Level Fusion Model (Walia et al., 2019)

Proposed Fingerprint $_{\mathrm{CNN}}$ \& Fingervein $_{\mathrm{CNN}} \&$ Face $_{\mathrm{CNN}}$
Time (ms)

130

580

69

1 


\section{Table 13 (on next page)}

The accuracy rate for proposed systems and different recognition biometric system results. 


\begin{tabular}{|c|c|}
\hline Algorithms & Accuracy (\%) \\
\hline Enhanced Fingerprint $_{\mathrm{CNN}}$ using (Cherrat, Alaoui \& Bouzahir, 2019) & 99.48 \\
\hline Enhanced Fingervein $_{\mathrm{CNN}}$ using (Ying, et al., 2017) & 99.53 \\
\hline Fingervein $_{\mathrm{CNN}}$ ( Itqan, et al., 2017) & 96.65 \\
\hline Proposed Face $_{\mathrm{CNN}}$ & 99.13 \\
\hline Proposed Fingerprint $_{\mathrm{CNN} \&}$ Fingervein ${ }_{\mathrm{CNN}}$ & 99.51 \\
\hline Proposed Fingerprint $_{\mathrm{CNN} \&}$ Face $_{\mathrm{CNN}}$ & 99.31 \\
\hline Proposed Fingervein $_{\mathrm{CNN} \&}$ Face $_{\mathrm{CNN}}$ & 99.33 \\
\hline Fingerprint $_{\mathrm{ANN}}$ \& Fingervein ${ }_{\mathrm{ANN}} \&$ Face $_{\mathrm{ANN}}($ Rajesh \& Selvarajan, 2017) & 99.23 \\
\hline Score Level Fusion Model (Walia et al., 2019) & 99.61 \\
\hline Proposed Fingerprint $_{\mathrm{CNN}} \&$ Fingervein $_{\mathrm{CNN}} \&$ Face $_{\mathrm{CNN}}$ & 99.49 \\
\hline
\end{tabular}

1 